Bull. Austral. Math. Soc.

VOL. 74 (2006) [185-196]

\title{
CHARACTERISATIONS OF $\Omega$-STABILITY AND STRUCTURAL STABILITY VIA INVERSE SHADOWING
}

\author{
Taeyoung Choi, Keonhee Lee and Yong Zhang
}

\begin{abstract}
We give characterisations of $\Omega$-stable diffeomorphisms and structurally stable diffeomorphisms via the notions of weak inverse shadowing and orbital inverse shadowing, respectively. More precisely, it is proved that the $C^{1}$ interior of the set of diffeomorphisms with the weak inverse shadowing property coincides with the set of $\Omega$-stable diffeomorphisms and the $C^{l}$ interior of the set of diffeomorphisms with the orbital inverse shadowing property coincides with the set of structurally stable diffeomorphisms.
\end{abstract}

\section{INTRODUCTION}

Structurally stable systems and $\Omega$-stable systems have been the main objects of interests in the global qualitative theory of dynamical systems over recent 30 years and various attempts have been made to characterise the systems via the notions of hyperbolicity, shadowing, Axiom A, strong transversality condition, no-cycle condition, et cetera.

It was proved in [10] and [11] that the $C^{\mathbf{1}}$ interior of the set of topologically stable diffeomorphisms [respectively, topologically stable $C^{1}$ vector fields] coincides with the set of structurally stable diffeomorphisms [respectively, structurally stable $C^{1}$ vector fields]. Sakai [14] showed that the $C^{1}$ interior of the set of diffeomorphisms having the shadowing property was characterised as the set of structurally stable diffeomorphisms.

The weak shadowing property which is really weaker than the shadowing property was introduced by Corless and Pilyugin [2], and they proved that the weak shadowing property is generic in the set of homoemorphisms on a $C^{\infty}$ closed manifold endowed with the $C^{0}$ topology. Moreover Sakai [16] showed that every element in the $C^{1}$ interior of the set of diffeomorphisms on a $C^{\infty}$ closed surface having the weak shadowing property is $\Omega$-stable, but the converse does not hold even in dimension 2 .

Recently Pilyugin, Rodionova and Sakai [13] proved that the $C^{1}$ interior of the set of diffeomorphisms having the orbital shadowing property consists of structurally stable diffeomorphisms.

Received 2nd March, 2006

This work was supported by the KRF Grant funded by the Korean Government (MOEHRD) (KRF2005-070-C00015).

Copyright Clearance Centre, Inc. Serial-fee code: 0004-9727/06 \$A2.00+0.00. 
The inverse shadowing property which is a "dual" notion of shadowing was introduced by Corless and Pilyugin in [2], and the qualitative theory of dynamical systems with the property was developed by various authors (see $[1,2,3,5,6,7,13]$, for example).

In this paper we introduce the notion of weak [respectively, orbital] inverse shadowing which is a "dual" notion of weak [respectively, orbital] shadowing, and prove that the $C^{1}$ interior of the set of diffeomorphisms with the weak [respectively, orbital] inverse shadowing property coincides with the set of $\Omega$-stable [respectively structurally stable] diffeomorphisms. These facts improve the results in $[6,13,16]$ which say that every structurally diffeomorphism has the inverse shadowing property with respect to classes of continuous methods, and the $C^{\mathbf{1}}$ interior of the set of diffeomorphisms satisfying persistency is characterised as the set of diffeomorphisms satisfying Axiom A and the strong transversality condition.

\section{Preliminaries}

Consider a dynamical system generated by a homeomorphism $f$ of a compact metric space $X$ with a metric $d$. For a point $x \in X$, we denote by $O(x, f)$ its orbit in the system $f:$ that is, the set

$$
O(x, f)=\left\{f^{n}(x): n \in \mathbb{Z}\right\} \text {. }
$$

We say that a sequence $\xi=\left\{x_{n} \in X: n \in \mathbb{Z}\right\}$ is a $\delta$-pseudo orbit of $f$ if the inequalities

$$
d\left(f\left(x_{n}\right), x_{n+1}\right)<\delta, n \in \mathbb{Z}
$$

hold. A $\delta$-pseudo orbit is a natural model of computer output in a process of numerical investigation of the system $f$. In this case, the value $\delta$ measures errors of the method, round-off errors, et cetera.

Recall that $f$ has the shadowing property if given $\varepsilon>0$ there exists $\delta>0$ such that for any $\delta$-pseudo orbit $\xi=\left\{x_{n}: n \in \mathbb{Z}\right\}$ we can find a point $y \in X$ with the property

$$
d\left(f^{n}(y), x_{n}\right)<\varepsilon, n \in \mathbf{Z} .
$$

Of course, if $f$ has the shadowing property formulated above, then the results of its numerical study with a proper accuracy reflect its qualitative structure.

It is said that $f$ has the weak shadowing property [respectively orbital shadowing property] if given $\varepsilon>0$ there exists $\delta>0$ such that for any $\delta$-pseudo orbit $\xi=\left\{x_{n}: n\right.$ $\in \mathbb{Z}\}$ of $f$ we can find a point $y \in X$ with the property

$$
\xi \subset N(\varepsilon, O(y, f))\left[\text { respectively } d_{H}(\bar{\xi}, \overline{O(y, f)})<\varepsilon\right],
$$

where $d_{H}$ denotes the Hausdorff distance on the set of compact subsets of $X$. The weak shadowing property was introduced in [2] and the orbital shadowing property was introduced in [14]. 
Let $X^{\mathbf{Z}}$ be the space of all two sided sequences $\xi=\left\{x_{n}: n \in \mathbb{Z}\right\}$ with elements $x_{n} \in X$, endowed with the product topology. For $\delta>0$, let $\Phi_{f}(\delta)$ denote the set of all $\delta$-pseudo orbits of $f$. A mapping $\varphi: X \rightarrow \Phi_{f}(\delta) \subset X^{\mathbf{Z}}$ is said to be a $\delta$-method for $f$ if $\varphi(x)_{0}=x$, where $\varphi(x)_{0}$ denotes the 0 -th component of $\varphi(x)$. Then each $\varphi(x)$ is a $\delta$-pseudo orbit of $f$ through $x$. For convenience, write $\varphi(x)$ for $\left\{\varphi(x)_{k}\right\}_{k \in \mathbf{Z}}$. Say that $\varphi$ is a continuous $\delta$-method for $f$ if the map $\varphi$ is continuous. The set of all $\delta$-methods [respectively, continuous $\delta$-methods] for $f$ will be denoted by $\mathcal{T}_{0}(f, \delta)$ [respectively $\mathcal{T}_{c}(f, \delta)$ ]. If $g: X \rightarrow X$ is a homeomorphism with $d_{0}(f, g)<\delta$, where

$$
d_{0}(f, g)=\sup _{x \in X}\left\{d(f(x), g(x)), d\left(f^{-1}(x), g^{-1}(x)\right)\right\},
$$

then $g$ induces a continuous $\delta$-method $\varphi_{g}$ for $f$ by defining

$$
\varphi_{g}(x)=\left\{g^{n}(x): n \in \mathbb{Z}\right\} .
$$

Let $\mathcal{T}_{h}(f, \delta)$ denote the set of all continuous $\delta$-methods $\varphi_{g}$ for $f$ which are induced by $g \in Z(X)$ with $d_{0}(f, g)<\delta$, where $Z(X)$ denotes the space of homeomorphisms on $X$ with the $C^{0}$ metric $d_{0}$. We define $\mathcal{T}_{\alpha}(f)$ by

$$
\mathcal{T}_{\alpha}(f)=\bigcup_{\delta>0} \mathcal{T}_{\alpha}(f, \delta)
$$

where $\alpha=0, c, h$. Clearly,

$$
\mathcal{T}_{h}(f) \subset \mathcal{T}_{c}(f) \subset \mathcal{T}_{0}(f) .
$$

Note that a method in $\mathcal{T}_{c}(f)$ need not be generated by a single mapping.

We say that $f$ has the shadowing property [respectively inverse shadowing property] with respect to the class $\mathcal{T}_{\alpha}, \alpha=0, c, h$, if for any $\varepsilon>0$ there is $\delta>0$ such that for any $\delta$-method $\varphi$ in $\mathcal{T}_{\alpha}(f, \delta)$ and any point $x \in X$ there exists a point $y \in X$ for which

$$
d\left(f^{n}(y), \varphi(x)_{n}\right)<\varepsilon\left[\text { respectively } d\left(f^{n}(x), \varphi(y)_{n}\right)<\varepsilon\right], n \in \mathbb{Z} .
$$

When we study the qualitative theory of differentiable dynamical systems (or diffeomorphisms) the notion of shadowing (or inverse shadowing) with respect to the class $\mathcal{T}_{c}$ (or $\mathcal{T}_{h}$ ) is sometimes too strong as can be seen in ([6, Theorem 3.3]). Here we give another types of shadowing and inverse shadowing as follows.

For our purpose, let $M$ be a $C^{\infty}$ closed $n$-dimensional manifold with metric $d$ induced by a Riemannian metric $\|\cdot\|$ on $T M$, and let Diff $(M)$ denote the space of $C^{1}$ diffeomorphisms on $M$ with the $C^{1}$ metric $d_{1}$. As before, for any $\delta>0$ and $f \in \operatorname{Diff}(M)$, every $g \in \operatorname{Diff}(M)$ with $d_{1}(f, g)<\delta$ induces a continuous $\delta$-method $\varphi_{g}: M \rightarrow M^{\mathbf{z}}$ for $f$ by defining

$$
\varphi_{g}(x)=\left\{g^{k}(x): k \in \mathbb{Z}\right\} .
$$


Let $\mathcal{T}_{d}(f, \delta)$ denote the set of all continuous $\delta$-methods $\varphi_{g}$ for $f$ which are induced by $g \in \operatorname{Diff}(M)$ with $d_{1}(f, g)<\delta$. Put

$$
\mathcal{T}_{d}(f)=\bigcup_{\delta>0} \mathcal{T}_{d}(f, \delta)
$$

Similarly we say that $f$ has the shadowing property [respectively, inverse shadowing property] with respect to the class $\mathcal{T}_{d}$ if for any $\varepsilon>0$ there is $\delta>0$ such that for any $\delta$-method $\varphi$ in $\mathcal{T}_{d}(f, \delta)$ and any point $x \in M$ there exists a point $y \in M$ for which

$$
d\left(f^{n}(y), \varphi(x)_{n}\right)<\varepsilon\left[\text { respectively } d\left(f^{n}(x), \varphi(y)_{n}\right)<\varepsilon\right], n \in \mathbb{Z} .
$$

Now let us recall the notion of weak [respectively, orbital] inverse shadowing which is a "dual" notion of weak [respectively, orbital] shadowing (see [1]).

DEFINITION 2.1: We say that $f$ has the weak [respectively, orbital] inverse shadowing property with respect to the class $\mathcal{T}_{\alpha}, \alpha=0, c, h, d$ if for any $\varepsilon>0$ there exists $\delta>0$ such that for any $\delta$-method $\varphi \in \mathcal{T}_{\alpha}(f, \delta)$ and any point $x \in M$ there is a point $y \in M$ for which

$$
\varphi(y) \subset N(\varepsilon, O(x, f))\left[\text { respectively, } d_{H}(\overline{O(x, f)}, \overline{\varphi(y)})<\varepsilon\right] .
$$

REMARK 2.2. An appropriate choice of the class of admissible pseudo orbits is crucial when we consider the notions of inverse shadowing (see $[\mathbf{3}, \mathbf{6}, \mathbf{1 1}])$. Suppose that $\mathcal{T}_{a}(f)$ $\subset \mathcal{T}_{b}(f)$ for some $f \in \operatorname{Diff}(M)$ and $a, b \in\{0, c, h, d\}$. If $f$ has the weak [respectively, orbital] inverse shadowing property with respect to the class $\mathcal{T}_{b}$ then it clearly has the weak [respectively, orbital] inverse shadowing property with respect to the class $\mathcal{T}_{a}$. We can easily show that every irrational rotation $f$ on the unit circle $S^{1}$ has the weak inverse shadowing with respect to the class $\mathcal{T}_{c}$ (or $\mathcal{T}_{h}$ ), but it does not have the inverse shadowing property with respect to the class $\mathcal{T}_{c}$ (or $\mathcal{T}_{h}$ ). Recently Choi, Kim and Lee [1] showed that the weak inverse shadowing property for $\mathcal{T}_{h}$ is generic in the space of homeomorphisms on a compact metric space with the $C^{0}$ topology.

\section{MAIN THEOREMS}

The aim of this paper is to investigate the dynamics of diffeomorphisms belonging to the $C^{1}$ interior of the set of diffeomorphisms having the weak and orbital inverse shadowing property with respect to the class $\mathcal{T}_{d}$. Through the paper, for simplicity, we shall abbreviate "weak [respectively orbital] inverse shadowing property with respect to the class $\mathcal{T}_{d}$ " to "weak [respectively, orbital] inverse shadowing property".

We say that $f \in \operatorname{Diff}(M)$ is structurally stable if there exists a $C^{1}$ neighbourhood $\mathcal{U}$ of $f$ in $\operatorname{Diff}(M)$ such that every $g \in \mathcal{U}$ is topologically conjugate to $f$. Let $\Omega(f)$ denote the set of nonwandering points of $f$. Recall that $f$ is $\Omega$-stable if there is a $C^{1}$ 
neighbourhood $\mathcal{V}$ of $f$ such that for any $g \in \mathcal{V}$ the restriction of $f$ to $\Omega(f)$ and $g$ to $\Omega(g)$ are topologically conjugate.

We denote by $\operatorname{WIS}(M)$ the set of $f \in \operatorname{Diff}(M)$ having the weak inverse shadowing property, and denote by $\operatorname{OIS}(M)$ the set of $f \in \operatorname{Diff}(M)$ having the orbital inverse shadowing property. The following two theorems are the main results of this paper.

ThEOREM 3.1. The $C^{1}$ interior of WIS $(M)$ in $\operatorname{Diff}(M)$ coincides with the set of $\Omega$-stable diffeomorphisms on $M$.

Theorem 3.2. The $C^{1}$ interior of $\operatorname{OIS}(M)$ in $\operatorname{Diff}(M)$ coincides with the set of structurally stable diffeomorphisms on $M$.

REMARK 3.3. It was proved in [16] that every element in the $C^{1}$ interior of the set of diffeomorphisms on a $C^{\infty}$ closed surface having the weak shadowing property is $\Omega$-stable, but the converse does not hold in general. We can see that the above result does not generalise to higher dimensions. In fact, it was proved in [9] that there is a $C^{1}$ open set $\mathcal{U}$ of the set of diffeomorphisms on the 3-torus such that every $f \in \mathcal{U}$ is topologically transitive and it is not Anosov. This means that every $f \in \mathcal{U}$ is not $\Omega$-stable but it has the weak shadowing property.

REMARK 3.4. Theorem 3.2 improves the results obtained by $[6,13,16]$. It was proved in [13] that every structurally stable diffeomorphism has the inverse shadowing property with respect to the class $\theta_{c}$ (or $\theta_{s}$ ). It was shown in [16] that the $C^{1}$ interior of the set of diffeomorphisms with persistency is characterised as the set of structurally stable diffeomorphisms. It was also proved in [6] that the $C^{1}$ interior of the set of diffeomorphisms having the inverse shadowing property with respect to the class $\mathcal{T}_{d}$ coincides with the set of structurally stable diffeomorphisms.

Note that the notion of the class $\theta_{c}$ in [13] is the same as that of the class $\mathcal{T}_{c}$ in this paper, and the notion of persistency in [16] is the same as that of the inverse shadowing property with respect to the class $\mathcal{T}_{h}$ in this paper.

If $f \in \operatorname{Diff}(M)$ has the inverse shadowing property with respect to the class $\mathcal{T}_{k}$ then it clearly has the inverse shadowing property with respect to the class $\mathcal{T}_{d}$, but the converse does not hold in general. Moreover if $f \in \operatorname{Diff}(M)$ has the inverse shadowing property with respect to the class $\mathcal{T}_{d}$ then it has the orbital inverse shadowing property, but the converse does not hold. Indeed, an irrational rotation on the unit circle has the orbital inverse shadowing property but does not have the inverse shadowing property with respect to the class $\mathcal{T}_{d}$.

We say that $f \in \operatorname{Diff}(M)$ is hyperbolic on a closed invariant set $\Lambda \subset M$ if there is a continuous splitting of the tangent bundle, $\left.T M\right|_{\Lambda}=E^{s} \oplus E^{u}$, and there are constants $C>0,0<\lambda<1$, such that

$$
\left\|\left.D f^{n}\right|_{E_{z}^{\ddagger}}\right\|<C \lambda^{n}, \quad\left\|\left.D f^{-n}\right|_{E_{x}^{\Sigma}}\right\|<C \lambda^{n}
$$


for any $n>0$ and $x \in \Lambda$. The stable [respectively unstable] manifold of $x \in \Lambda$ is defined by $W^{s}(x, f)$ [respectively $W^{u}(x, f)$ ], which is the set of points $p \in M$ such that $d\left(f^{k}(x), f^{k}(p)\right)$ tends to 0 as $k$ tends to $\infty$ [respectively $-\infty$ ]. We say that $f$ satisfies Axiom $A$ if its periodic points are dense in the set of nonwandering points $\Omega(f)$, and $f$ is hyperbolic on $\Omega(f)$. An Axiom A diffeomorphism $f$ is said to satisfy the strong transversality condition if $W^{s}(x, f)$ and $W^{u}(y, f)$ are transversal for any $x, y \in \Omega(f)$.

Let $\mathcal{F}(M)$ be the set of $f \in \operatorname{Diff}(M)$ having a $C^{1}$ neighbourhood $\mathcal{U} \subset \operatorname{Diff}(M)$ such that for $g \in \mathcal{U}$, every periodic point of $g$ is hyperbolic. Hayashi [4] proved that $\mathcal{F}(M)$ is contained in the set of diffeomorphisms satisfying Axiom A with no-cycle condition. It is well known that $f$ is $\Omega$-stable if and only if it satisfies Axiom $A$ and no-cycle condition.

\section{Proof of Theorem 3.1}

First we show that every element $f$ in the $C^{1}$ interior of $\operatorname{WIS}(M)$ in $\operatorname{Diff}(M)$, WIS $(M)^{0}$, belongs to $\mathcal{F}(M)$. Then it is $\Omega$-stable by the result of Hayashi [4]. To get a contradiction, we assume that there exists $f \in \operatorname{WIS}(M)^{0} \backslash \mathcal{F}(M)$. Choose a $C^{1}$ neighbourhood $\mathcal{U}$ of $f$ in WIS $(M)^{0}$ and take a diffeomorphism $f^{\prime} \in \mathcal{U}$ having a nonhyperbolic periodic point $p$. Let $m$ be the period of the point $p$. Passing from $f^{\prime}$ to its $C^{1}$ small perturbation $f^{\prime \prime}$, we may assume that the derivative $D\left(f^{\prime \prime}\right)^{m}(p)$ has an eigenvalue equal to 1 .

Standard arguments (as in the proof of [14, Theorem 3.2]) enable us to find a $C^{1}$ small perturbation of $f$ in $\mathcal{U}$ (again denoted by $f$ ) having the following properties:

(1) $p$ is a periodic point of $f$ with period $m$ (we denote $p_{i}=f^{i}(p)$ for $i=0, \ldots, m)$;

(2) if $\lambda_{1}, \ldots, \lambda_{n}$ are the eigenvalues of the derivative $D f^{m}(p)$, then $\lambda_{1}=1$ and $\left|\lambda_{j}\right| \neq 1$ for $j=2, \ldots, n$;

(3) we can introduce local coordinates $y=\left(y_{1}, \ldots, y_{n}\right)$ in disjoint neighbourhoods $U_{i}$ of the points $p_{i}$ so that $p_{i}$ is the origin in $U_{i}$, and

(3.1) if $L_{0}$ is the subspace of the tangent space $T_{p} M$ (identified with $\mathbb{R}^{n}$ ) corresponding to the eigenvalue $\lambda_{1}$ of $D f^{m}(p)$, then the spaces

$$
L^{i}=D f^{m}(p) L_{0}, i=0, \ldots, m-1,
$$

coincide with the subspaces

$$
\left\{y \in \mathbb{R}^{n}: y_{2}=\cdots=y_{n}=0\right\}
$$

in the coordinates of the corresponding neighbourhoods;

(3.2) there is a number $a>0$ such that the mapping $\varphi^{(i)}$, the restriction of $f$ to the set $U_{i}^{\prime}=U_{i} \cap\{|y|<4 a\}$, maps $U_{i}^{\prime}$ into $U_{i+1}$ (of course, 


$$
\begin{aligned}
& \left.U_{m}=U_{0}\right) \text { and is given by the formula } \\
& \qquad \varphi^{(i)}(y)=\left(k_{i} y_{1}, B_{i} y^{\prime}\right),
\end{aligned}
$$

where $y^{\prime}=\left(y_{2}, \ldots, y_{n}\right)$.

Since $f$ is a diffeomorphism, we have $k_{i} \neq 0$ for $i=0, \ldots, m-1$. It follows from (2), (3.1) and (3.2) that $k_{0} k_{1} \ldots k_{m-1}=1$ and the matrix $B=B_{m-1} \ldots B_{0}$ is hyperbolic. Let

$$
\begin{aligned}
& K_{1}=\min _{0 \leqslant i \leqslant m-1}\left|k_{i} k_{i-1} \ldots k_{0}\right|, \\
& K_{2}=\max _{0 \leqslant i \leqslant m-1}\left|k_{i} k_{i-1} \ldots k_{0}\right|, \text { and } K=\frac{K_{1}}{K_{2}} .
\end{aligned}
$$

It is easy to construct a diffeomorphism $g_{\lambda} \in \mathcal{U}$ coinciding with $f$ outside $U_{m-1}$ such that $\psi_{\lambda}(y)$, the restriction of $g_{\lambda}$ to the set $U_{m-1}^{\prime}$, is given by the formula

$$
\psi_{\lambda}(y)=\left(\lambda y_{1}, B_{m-1} y^{\prime}\right)
$$

where $0<k_{0} k_{1} \ldots k_{m-2} \lambda=\mu<1$ and $1-\mu$ is arbitrary small. Fix $\varepsilon=a K$, and find a corresponding $\delta>0$ from the definition of $f \in$ WIS $(M)^{0}$. Take a diffeomorphism $g_{\lambda} \in \mathcal{U}$ with $d_{1}\left(f, g_{\lambda}\right) \leqslant \delta$, and let

$$
x_{0}=\left(\frac{2 a}{K_{2}}, 0, \ldots, 0\right) .
$$

Note that for any point $f^{j}\left(x_{0}\right) \in O\left(x_{0}, f\right)$, its first coordinate $f^{j}\left(x_{0}\right)_{1}$ is obtained by

$$
k_{i-1} \ldots k_{0} \frac{2 a}{K_{2}}
$$

for some $i$ with $j \equiv i(\bmod m)$, and satisfies the inequalities

$$
2 \varepsilon=K_{1} \frac{2 a}{K_{2}} \leqslant\left|f^{j}\left(x_{0}\right)_{1}\right| \leqslant K_{2} \frac{2 a}{K_{2}}=2 a .
$$

Formula (4.1) implies that for any point $\left(y_{1}, y^{\prime}\right)$ with $y^{\prime} \neq 0$, its trajectory leaves the set $N\left(\varepsilon, O\left(x_{0}, f\right)\right)$. Hence the inclusion

$$
O\left(y, g_{\lambda}\right) \subset N\left(\varepsilon, O\left(x_{0}, f\right)\right)
$$

shows that $y=\left(y_{1}, 0, \ldots, 0\right)$. If $y_{1}=0$, then we get

$$
(0,0, \ldots, 0) \notin N\left(\varepsilon, O\left(x_{0}, f\right)\right) .
$$

If $y_{1} \neq 0$, there exists $j_{0} \in \mathbf{N}$ such that $\mu^{m j_{0}}<\varepsilon$, This implies

$$
g_{\lambda}^{m j_{0}}\left(y_{1}, 0, \ldots, 0\right)=\left(\mu^{m j_{0}}, 0, \ldots, 0\right) \notin N\left(\varepsilon, O\left(x_{0}, f\right)\right),
$$

and so contradict to the fact that $f \in \operatorname{WIS}(M)^{0}$. 
Next we show that every $\Omega$-stable diffeomorphism has the weak inverse shadowing property. Suppose that $f \in \operatorname{Diff}(M)$ is $\Omega$-stable, and let $\Omega_{1}, \ldots, \Omega_{m}$ be the basic sets of $f$ with

$$
\Omega(f)=\Omega_{1} \cup \cdots \cup \Omega_{m} .
$$

Let $\alpha=\min \left\{d\left(\Omega_{i}, \Omega_{j}\right): i \neq j\right\}$ and $0<\varepsilon<\alpha / 4$. Since each $\Omega_{i}$ is a closed hyperbolic invariant set for $f$, we can choose a $C^{\mathbf{1}}$ neighbourhood $\mathcal{U}_{i}$ of $f$ in $\operatorname{Diff}(M)$ and a continuous $\operatorname{map} \Phi_{i}: \mathcal{U}_{i} \rightarrow C^{0}\left(\Omega_{i}, M\right)$ satisfying

(1) $\Phi_{i}(f)\left(\Omega_{i}\right)=\Omega_{i}$;

(2) $\Phi_{i}(g)\left(\Omega_{i}\right)$ is a hyperbolic invariant set for $g, g \in \mathcal{U}_{i}$;

(3) $\Phi_{i}(g)$ is a homeomorphism of $\Omega_{i}$ onto $\Phi_{i}(g)\left(\Omega_{i}\right)$ and topologically conjugates the restriction of $f$ to $\Omega_{i}$ to the restriction of $g$ to $\Phi_{i}(g)\left(\Omega_{i}\right)$;

(4) there is a constant $K_{i}$ such that

$$
d_{0}\left(\Phi_{i}(g), \Phi_{i}(f)\right)<K_{i} d_{0}(g, f),
$$

where $i=1,2, \ldots, m$.

Choose $\delta_{i}>0$ satisfying $B_{d_{1}}\left(\delta_{i}, f\right) \subset \mathcal{U}_{i}$ for each $i$, and let

$$
\delta=\min _{1 \leqslant i \leqslant m}\left\{\delta_{i}, \frac{\varepsilon}{4 K_{i}}\right\}
$$

where

$$
B_{d_{1}}\left(\delta_{i}, f\right)=\left\{g \in \operatorname{Diff}(M): d_{1}(f, g)<\delta_{i}\right\} .
$$

Let $g \in B_{d_{1}}(\delta, f)$ and $x \in M$. Suppose $\omega(x, f) \subset \Omega_{i}$ for some $i$ and take a point $z \in \omega(x, f)$. Let $y=\Phi_{i}(g)(z)$. Then we have

$$
O(y, g) \subset N(\varepsilon, O(z, f)) \subset N(\varepsilon, \omega(x, f)) \subset N(\varepsilon, O(x, f)) .
$$

This means that $f$ has the weak inverse shadowing property, and so completes the proof of Theorem 3.1.

\section{Proof of Theorem 3.2}

It is well known that the $C^{1}$ interior of the Kupka-Smale diffeomorphisms consists of structurally stable diffeomorphisms, and we see that every structurally stable diffeomorphism has the orbital inverse shadowing property by [12, Theorem 1.1].

To show that every element $f$ in the $C^{1}$ interior of $\operatorname{OIS}(M)$ in $\operatorname{Diff}(M), \operatorname{OIS}(M)^{0}$, is structurally stable, it suffices to show that $f$ has the strong transversality condition by applying the first part of the proof of Theorem 3.1. That is, it is enough to prove that if $p$ and $q$ are periodic points of $f \in \operatorname{OIS}(M)^{0}$ then their stable manifold $W^{s}(p, f)$ and unstable manifold $W^{u}(q, f)$ are transverse. To get a contradiction, we assume that 
there is a diffeomorphism $f \in \operatorname{OIS}(M)^{0}$, having periodic points $p$ and $q$ and a point $r$ of nontransverse intersection of $W^{s}(p, f)$ and $W^{u}(q, f)$. Note that the point $r$ is wandering, and $p$ and $q$ are in different basic sets. To simplify presentation, we assume that $p$ and $q$ are fixed points. (The case of periodic point is considered similarly.) By the same techniques described in the proof of [13, Theorem 4.1], for any $C^{1}$ neighbourhood $\mathcal{U}$ of $f$, we can choose a $C^{1}$ small perturbation of $f$ (denoted by $f$ ) with the following properties:

(1) $p$ and $q$ are fixed points of $f$;

(2) $f$ is linear in a neighbourhood of $p$;

(3) $r$ is a point of nontransverse intersection of $W^{s}(p, f)$ and $W^{u}(q, f)$;

(4) there exists a small open (with respect to the inner topology of $W^{u}(q, f)$ ) disk $\mathcal{C}^{u}\left(r^{\prime}\right) \subset W^{u}(q, f)$ of $r^{\prime}$ such that $\mathcal{C}^{u}(r)=f\left(\mathcal{C}^{u}\left(r^{\prime}\right)\right) \subset L^{u}+r$, where $r^{\prime}=f^{-1}(r)$ and $L^{u}=T_{r} W^{u}(q, f)$.

Let $\Omega_{1}, \ldots, \Omega_{m}$ be the basic sets of $f$ with

$$
\Omega(f)=\Omega_{1} \cup \cdots \cup \Omega_{m},
$$

and suppose that $q \in \Omega_{1}$ and $p \in \Omega_{2}$. Since the point $r$ is wandering, there is a neighbourhood $V$ of $r$ such that $f^{n}(V) \cap f^{m}(V)=\emptyset$ for all $n \neq m$.

We first claim that there is a neighbourhood $U(\subset V)$ of $r$ such that for each $x \in U$,

$$
x \notin W^{u}\left(\Omega_{2}\right) \cap W^{s}\left(\Omega_{1}\right) .
$$

Since $f$ satisfies Axiom A and no cycle condition, there exists a continuous Lyapunov function $L: M \rightarrow \mathbb{R}$ such that

(1) $L(f(x))<L(x)$ for $x \in M \backslash \Omega(f)$;

(2) $L$ is constant on $\Omega_{i}, i=1, \ldots, m$.

Since $L(p)<L(r)<L(q)$ and $L$ is continuous, there is a neighbourhood $U(\subset V)$ of $r$ such that for all $x \in U$,

$$
L(p)<L(x)<L(q)
$$

Then the set $U$ satisfies (5.1).

Next we show that there exist $\varepsilon^{\prime}>0$ and $\delta^{\prime}>0$ such that

(1) $N\left(\delta^{\prime}, r^{\prime}\right) \subset U^{\prime}$, where $U^{\prime}=f^{-1}(U)$;

(2) for any $x \in N\left(\delta^{\prime}, r^{\prime}\right) \backslash \mathcal{C}^{u}\left(r^{\prime}\right)$, we can find $l \geqslant 1$ satisfying

$$
d\left(f^{-l}(x), \overline{O(r, f)}\right) \geqslant \varepsilon^{\prime} .
$$

To show this, we take $\varepsilon_{1}>0$ satisfying $\varepsilon_{1} \leqslant d\left(\Omega_{1}, \Omega_{2}\right)$. Choose $0<\varepsilon_{2}<\varepsilon_{1} / 4$. Let

$$
O_{q}(r)=O(r, f) \cap N\left(\varepsilon_{2}, q\right) \text { and } O_{p}(r)=O(r, f) \backslash N\left(\varepsilon_{2}, q\right) .
$$


Note that $d\left(O_{p}(r), \Omega_{1}\right)>0$. Choose $\varepsilon_{3}<\varepsilon_{2}$ satisfying

$$
2 \varepsilon_{3} \leqslant d\left(O_{p}(r), \Omega_{1}\right)
$$

For the constant $\varepsilon_{3}$, there exists $\delta_{1}>0$ and $l_{1} \geqslant 1$ such that for any $x \in N\left(\delta_{1}, r^{\prime}\right) \cap W^{u}\left(\Omega_{1}\right)$,

$$
f^{-l}(x) \in N\left(\varepsilon_{3}, \Omega_{1}\right)
$$

for all $l \geqslant l_{1}$. For this $l_{1}$, we take $0<\delta^{\prime}<\delta_{1}$ such that for any $x \in N\left(\delta^{\prime}, r^{\prime}\right) \backslash \mathcal{C}^{u}\left(r^{\prime}\right)$, there exists $l=l\left(\delta^{\prime}, x\right) \geqslant l_{1}$ satisfying

$$
f^{-l}(x) \notin N\left(2 \varepsilon_{2}, q\right)
$$

(This is from the stable manifold theorem). It is easy to find $\varepsilon_{4}>0$ satisfying

$$
d\left(\overline{O(r, f)}, \Omega_{i}\right)>\varepsilon_{4}
$$

for $i \neq 1,2$.

Let $\varepsilon^{\prime}=\min \left\{\varepsilon_{4}, \varepsilon_{3}\right\}$, and take $x \in N\left(\delta^{\prime}, r^{\prime}\right) \backslash \mathcal{C}^{u}\left(r^{\prime}\right)$. Then we can show that if $x \in W^{u}\left(\Omega_{i}\right)$ for $i \neq 1,2$, there exists $l \geqslant 1$ such that

$$
d\left(f^{-l}(x), \overline{O(r, f)}\right) \geqslant \varepsilon_{4} \geqslant \varepsilon^{\prime}
$$

if $x \in W^{u}\left(\Omega_{1}\right)$, there exists $l \geqslant l_{1}$ such that

$$
f^{-l}(x) \notin N\left(2 \varepsilon_{2}, q\right)
$$

So we have

$$
d\left(f^{-l}(x), O_{q}(r)\right)>\varepsilon^{\prime}
$$

for some $l \geqslant l_{1}$. Since $2 \varepsilon_{3}<d\left(\Omega_{1}, O_{p}(r)\right)$, we get

$$
d\left(f^{-l}(x), O_{p}(r)\right)>\varepsilon^{\prime}
$$

Moreover we have $f^{-l}(x) \in N\left(\varepsilon_{3}, \Omega_{1}\right)$. Combining these two statements, we obtain the inequality (5.2).

Let $\mathcal{C}^{s}(r)$ be the connected component of $W^{s}(p, f)$ contained in $U$ which contains $r$. By the same techniques as above, we can find $\varepsilon>0$ and $\delta>0$ such that

(1) $N(\delta, r) \subset U$;

(2) for any $x \in N(\delta, r) \backslash \mathcal{C}^{s}(r)$, there exists $l \geqslant 1$ satisfying

$$
d\left(f^{l}(x), \overline{O(r, f)}\right) \geqslant \varepsilon
$$


Now we show that $f$ does not have the orbital inverse shadowing property. It is easy to construct a $C^{1}$ small perturbation $g$ of $f$ such that

$$
\begin{aligned}
& g=f \quad \text { on } \quad M \backslash U^{\prime}, \quad g\left(U^{\prime}\right)=U, \quad \text { and } \\
& g\left(N\left(\delta^{\prime}, r^{\prime}\right) \cap \mathcal{C}^{u}\left(r^{\prime}\right)\right) \cap \mathcal{C}^{s}(r)=\emptyset .
\end{aligned}
$$

Let $\bar{\varepsilon}=\min \left\{\delta, \delta^{\prime}, \varepsilon / 2, \varepsilon^{\prime} / 2\right\}$. Then the proof is completed by showing that there is no point $x \in M$ satisfying

$$
d_{H}(\overline{O(x, g)}, \overline{O(r, f)}) \leqslant \bar{\varepsilon} .
$$

Suppose that there exists a point $x \in M$ satisfying

$$
d_{H}(\overline{O(x, g)}, \overline{O(r, f)}) \leqslant \bar{\varepsilon}
$$

Since $U$ is wandering for $g$, we can see that the set $O(x, g) \cap N(\delta, r)$ contains only one point, say $z$; and the set $O(x, g) \cap N\left(\delta^{\prime}, r^{\prime}\right)$ contains only one point, say $z^{\prime}$. Then we have

$$
g\left(z^{\prime}\right)=z, O^{+}(z, f)=O^{+}(z, g) \text { and } O^{-}\left(z^{\prime}, f\right)=O^{-}\left(z^{\prime}, g\right) .
$$

The inequalities (5.2) and (5.3) imply that

$$
z^{\prime} \in N\left(\delta^{\prime}, r^{\prime}\right) \cap \mathcal{C}^{u}\left(r^{\prime}\right) \text { and } z \in N(\delta, r) \cap \mathcal{C}^{s}(r) .
$$

This contradicts to (5.4), and so completes the proof.

\section{REFERENCES}

[1] T. Choi, S. Kim and K. Lee, 'Weak inverse shadowing and genericity', Bull. Korean Math. Soc. 43 (2006), 43-52.

[2] R. Corless and S. Pilyugin, 'Approximate and real trajectories for generic dynamical systems', J. Math. Anal. Appl. 188 (1995), 409-423.

[3] P. Diamond, K. Lee and Y. Han, 'Bishadowing and hyperbolicity', Internat. J. Bifur. Chaos appl. Sci. Engry. 12 (2002), 1779-1788.

[4] S. Hayashi, 'Diffeomorphisms in $F^{1}(M)$ satisfy Axiom A', Ergodic Theory Dynamical Systems 12 (1992), 233-253.

[5] P. Kloeden, J. Ombach and A. Porkrovskii, 'Continuous and inverse shadowing', Funct. Differ. Equ. 6 (1999), 137-153.

[6] K. Lee, 'Continuous inverse shadowing and hyperbolicity', Bull. Austral. Math. Soc. 67 (2003), 15-26.

[7] K. Lee and J. Park, 'Inverse shadowing of cirlce maps', Bull. Austral. Math. Soc. 69 (2004), 353-359.

[8] K. Lee and K. Sakai, 'Various shadowing properties and their equivalence', Disc. Contin. Dyn. Syst. 13 (2005), 533-539.

[9] R. Mañé, 'Contribution to stability conjecture', Topology 17 (1978), 383-396. 
[10] K. Moriyasu, 'The topological stability of diffeomorphisms', Nagoya Math. J. 123 (1991), 91-203.

[11] K. Moriyasu, K. Sakai and N. Sumi, 'Vector fields with topological stability', Trans. Amer. Math. Soc. 353 (2001), 3391-3408.

[12] S. Pilyugin, 'Inverse shadowing by continuous methods', Disc. Contin. Dyn. Syst. 8 (2002), 29-38.

[13] S. Pilyugin, A. Rodionova and K. Sakaj, 'Orbital and weak shadowing properties', Disc. Contin. Dyn. Sys. 9 (2003), 287-308.

[14] K. Sakai, 'Pseudo orbit tracing property and strong transversality of diffeomorphisms on closed manifolds', Osaka J. Math. 31 (1994), 373-386.

[15] K. Sakai, 'Diffeomorphism with persistency', Proc. Amer. Math. Soc. 124 (1996), 2249-2254.

[16] K. Sakai, 'Diffeomorphism with weak shadowing', Fund. Math. 168 (2001), 57-75.

Department of Mathematics

Chungnam National University

Daejeon, 305-764

Korea

e-mail: shadowcty@hanmail.net

khlee@math.cnu.ac.kr
Department of Mathematics

Suzhou University

Suzhou

China

e-mail: yongzhang@suda.edu.cn 